\title{
Low Striatal Dopamine D2-type Receptor Availability is Linked to Simulated Drug Choice in Methamphetamine Users
}

\author{
Scott J Moeller, ${ }^{*, 1,2}$, Kyoji Okita ${ }^{3,4}$, Chelsea L Robertson ${ }^{3,4}$, Michael E Ballard ${ }^{5,6}$, Anna B Konova ${ }^{7}$, \\ Rita Z Goldstein', Mark A Mandelkern ${ }^{4}$ and Edythe D London ${ }^{*, 1,3,4}$
}

'Departments of Psychiatry and Neuroscience, Icahn School of Medicine at Mount Sinai, New York, NY, USA; '2Department of Psychiatry, Stony Brook University School of Medicine, Stony Brook, NY, USA; ${ }^{2}$ Departments of Psychiatry and Biobehavioral Sciences, and Molecular and Medical Pharmacology, University of California Los Angeles (UCLA), Los Angeles, CA, USA; ${ }^{4}$ Veterans Administration of Greater Los Angeles (VAGLA), Los Angeles, CA, USA; ${ }^{5}$ University of California San Francisco, San Francisco, CA, USA; ${ }^{6}$ Veterans Administration Northern California Health Care System, Mather, CA, USA; ${ }^{7}$ Center for Neural Science, New York University, New York, NY, USA

Individuals with drug use disorders seek drugs over other rewarding activities, and exhibit neurochemical deficits related to dopamine, which is involved in value-based learning and decision-making. Thus, a dopaminergic disturbance may underpin drug-biased choice in addiction. Classical drug-choice assessments, which offer drug-consumption opportunities, are inappropriate for addicted individuals seeking treatment or abstaining. Fifteen recently abstinent methamphetamine users and 15 healthy controls completed two laboratory paradigms of 'simulated' drug choice (choice for drug-related vs affectively pleasant, unpleasant, and neutral images), and underwent positron emission tomography measurements of dopamine D2-type receptor availability, indicated by binding potential $\left(\mathrm{BP}_{\mathrm{ND}}\right)$ for $\left[{ }^{18} \mathrm{~F}\right]$ fallypride. Thirteen of the methamphetamine users and 10 controls also underwent $\left[{ }^{1} \mathrm{C}\right] \mathrm{NNCl} I 2$ PET scans to measure dopamine DI-type receptor availability. Group analyses showed that, compared with controls, methamphetamine users chose to view more methamphetamine-related images on one task, with a similar trend on the second task. Regression analyses showed that, on both tasks, the more methamphetamine users chose to view methamphetamine images, specifically vs pleasant images (the most frequently chosen images across all participants), the lower was their D2-type $\mathrm{BP}_{\mathrm{ND}}$ in the lateral orbitofrontal cortex, an important region in value-based choice. No associations were observed with $\mathrm{D} 2$-type $\mathrm{BP}_{\mathrm{ND}}$ in striatal regions, or with $\mathrm{DI}$-type $\mathrm{BP}_{\mathrm{ND}}$ in any region. These results identify a neurochemical correlate for a laboratory drug-seeking paradigm that can be administered to treatment-seeking and abstaining drug-addicted individuals. More broadly, these results refine the central hypothesis that dopamine-system deficits contribute to drug-biased decision-making in addiction, here showing a role for the orbitofrontal cortex.

Neuropsychopharmacology (20 I8) 43, 75 I-760; doi: I0.1038/npp.20 I7.I38; published online 26 July 2017

\section{INTRODUCTION}

Dopaminergic neurotransmission exerts considerable influence over a wide range of cognitive and behavioral processes, and deficient dopamine signaling is thought to underlie key pathological features of many neuropsychiatric disorders, including addictions. In this regard, positron emission tomography (PET) studies have demonstrated that chronic methamphetamine use is associated with below-control levels of D2-type (D2 and D3 not distinguished) receptor

*Correspondence: Dr SJ Moeller, Psychiatry and Neuroscience, Icahn School of Medicine at Mount Sinai, The Leon and Norma Hess Center for Science and Medicine, One Gustave L. Levy Place, Box 1230, New York, NY 10029-6574, USA, Tel: +212-241-6231, Fax: +212803-6743, E-mail: scott.moeller@stonybrookmedicine.edu or Dr ED London, Semel Institute of Neuroscience and Human Behavior, University of California Los Angeles, 760 Westwood Plaza, Los Angeles, CA 90024-1759, USA, Tel: +310-825-0606, Fax:+310-8250812, E-mail: elondon@mednet.ucla.edu

Received 12 December 2016; revised 20 June 2017; accepted 25 June 2017; accepted article preview online 30 June 2017 availability in the striatum (London et al, 2015). These deficits have been linked to indices of behavioral dysregulation, such as heightened impulsivity (Lee et al, 2009) and steep discounting of delayed rewards (Ballard et al, 2015). However, although these associations are important for understanding addiction phenomenology, they remain an important step removed from the actual choice to use drugs. Drug choice entails an active decision-making process that depends on a number of variables, such as value, availability, and the cost of the relevant alternatives (Padoa-Schioppa, 2011). Moreover, diagnostic criteria for substance-use disorders are largely defined by behaviors relevant to drugseeking and drug-taking, rather than to general behavioral dysregulation.

A useful paradigm for assessing drug-seeking and drugtaking in the human laboratory is the drug-choice procedure (Moeller and Stoops, 2015). Drug-choice procedures provide direct comparisons of an individual's propensity for selecting a drug in preference to an alternate palatable reward (eg, money, chocolate) (Greenwald et al, 2014; Hogarth 
and Chase, 2011). Members of our group have adapted this standard drug-choice procedure for use in abstinent or treatment-seeking individuals (who usually are prohibited from self-administering actual drugs in the laboratory), by replacing choices between tangible reinforcers (eg, drugs $v s$ money) with choices between more abstract/simulated reinforcers (ie, drug images $v s$ affectively pleasant images). In prior work, chronic cocaine users chose to view more cocaine-related images, and fewer pleasant images, than healthy control participants (Moeller et al, 2009); this heightened drug-related choice (specifically, the difference between drug choice and pleasant choice) correlated with recent and prospective drug use outside the laboratory (Moeller et al, 2013a).

Importantly, drug-related choice, whether tested using real or simulated rewards, has been associated with markers of dopaminergic neurotransmission. For example, cocaine users exhibited negative associations between preference for actual cocaine over money and amphetamine-induced dopamine release in the ventral striatum, assessed using PET with $\left[{ }^{11} \mathrm{C}\right]$ raclopride (Martinez et al, 2007), and dopamine D1-type receptor availability in the limbic striatum, assessed using PET with $\left[{ }^{11} \mathrm{C}\right] \mathrm{NNC112}$ (Martinez et al, 2009). Moreover, cocaine abusers who were carriers of a variant of the dopamine transporter gene (DAT1) that has been linked to more cue-reactive behavior exhibited greater simulated drug-choice than non-carriers (Moeller et al, 2013b).

The current study had two goals. The first was to examine simulated drug-choice behavior in methamphetamine users, a population that has not been tested with these tasks before. The second was to determine whether simulated methamphetamine choice is correlated with D2- and/or D1-type dopamine receptor availability, measured as binding potential $\left(\mathrm{BP}_{\mathrm{ND}}\right)$ of these receptor subtypes for $\left[{ }^{18} \mathrm{~F}\right]$ fallypride and $\left[{ }^{11} \mathrm{C}\right]$ NNC 112 , respectively. We hypothesized that chronic methamphetamine users would make more choices for methamphetamine-related images and fewer choices for pleasant images than healthy controls. We further hypothesized that, among the methamphetamine users, the choice for methamphetamine-related over pleasant images would be negatively associated with $\mathrm{D} 2$ - and D1-type $\mathrm{BP}_{\mathrm{ND}}$. For the latter hypothesis, prior work guided us to focus primarily on regions of the striatum, although we also explored correlations in other regions relevant to valuation and choice behavior, such as the orbitofrontal cortex (OFC)/ventromedial prefrontal cortex (PFC) and anterior cingulate cortex (ACC) (Bartra et al, 2013).

\section{MATERIALS AND METHODS}

\section{Participants}

Methamphetamine-dependent and healthy-control participants (15/group) provided written informed consent, as approved by the institutional review boards of UCLA and VAGLA. Eligibility was determined using self-reports, physical examination, and the Structured Clinical Interview for DSM-IV (First et al, 1996). Exclusion criteria were: CNS, cardiovascular, pulmonary, hepatic, or systemic disease; HIV seropositivity; pregnancy; English non-fluency; current psychotropic medication use; current Axis-I disorders other than nicotine dependence (both groups) or methamphetamine dependence or cannabis abuse or dependence (methamphetamine group). Methamphetamine-group participants were currently methamphetamine-dependent, and two were cannabis-dependent. Twenty-three participants smoked cigarettes (Table 1). Negative urine samples were required to exclude recent illicit drug use and pregnancy.

\section{Picture-Choice Tasks}

Two picture-choice tasks were adapted for methamphetamine users and administered before PET on the day of D2type receptor assay. These two tasks tap into different aspects of choice behavior (see Supplementary Information for discussion), and this difference can be viewed as a strength to facilitate testing of complementary associations with $\mathrm{BP}_{\mathrm{ND}}$. Images in three categories were selected from the International Affective Image System (IAPS) (Lang et al, 2008): pleasant (eg, smiling babies), unpleasant (eg, mutilation), and neutral (eg, household items) images. Another image category depicted methamphetamine-related images, matched to the IAPS images on size and ratio of human/

Table I Demographic Characteristics of Research Participants

\begin{tabular}{|c|c|c|c|}
\hline & Test & Controls $(N=15)$ & MA users $(N=15)$ \\
\hline Sex (women/men) & $\chi_{N=30}^{2}=1.22$ & $8 / 7$ & $5 / 10$ \\
\hline Ethnicity (Caucasian, African American, Other) & $\chi^{2} N=30=0.90$ & $6 / 5 / 4$ & $6 / 3 / 6$ \\
\hline Age (years) & $t_{28}=1.19$ & $32.6 \pm 7.4$ & $36.5 \pm 10.1$ \\
\hline Education & $t_{28}=0.41$ & $12.8 \pm 1.3$ & $12.5 \pm 2.9$ \\
\hline Daily tobacco/nicotine user (yes/no) & $\chi_{N=30}^{2}=0.19$ & $1 \mid / 4$ & $12 / 3$ \\
\hline Cigarettes per day (daily smokers only) & $t_{21}=1.59$ & $11.8 \pm 9.0$ & $6.8 \pm 6.0$ \\
\hline Age first MA use (years) & - & - & $23.7 \pm 8.8$ \\
\hline Frequency of MA use (days in last 30 days) & - & - & $25.5 \pm 7.2$ \\
\hline Intensity of MA use (grams per use) & - & - & $0.6 \pm 0.3$ \\
\hline MA abstinence (days) & - & - & $10.5 \pm|4|$. \\
\hline
\end{tabular}

Abbreviation: MA, methamphetamine.

Note: Numbers are frequency or mean \pm standard deviation. 


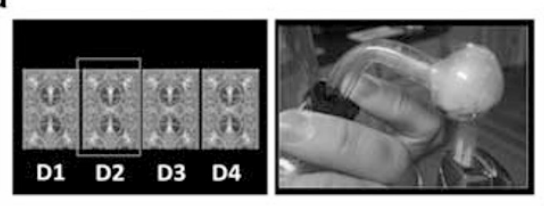

c

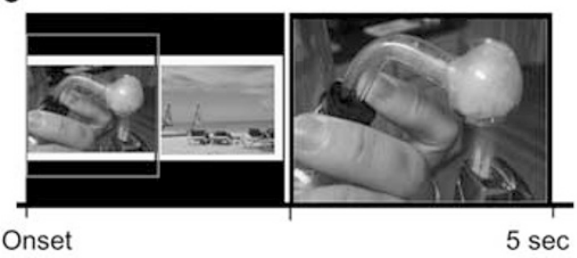

b

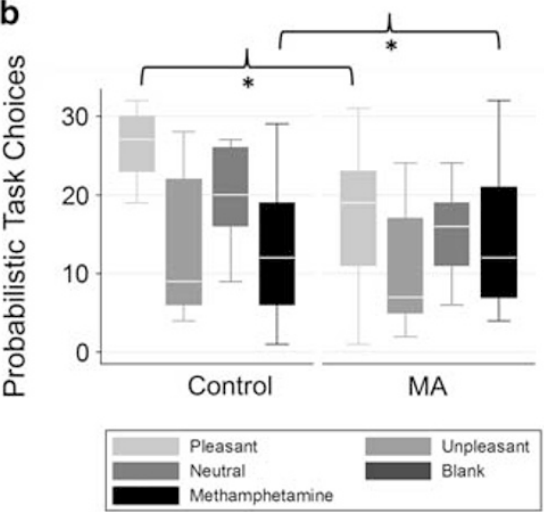

d

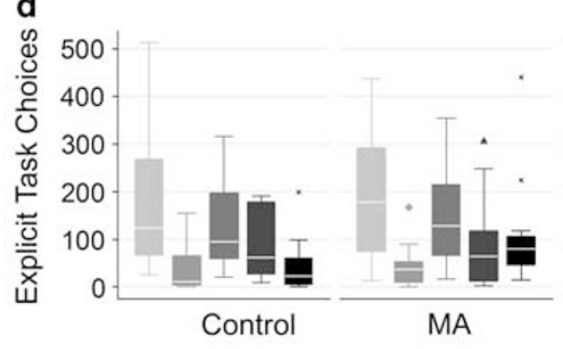

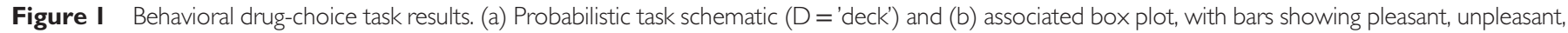

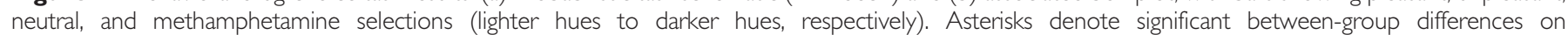

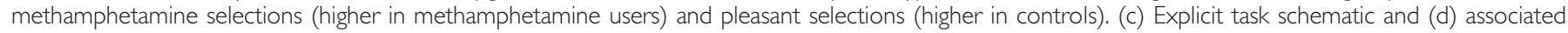

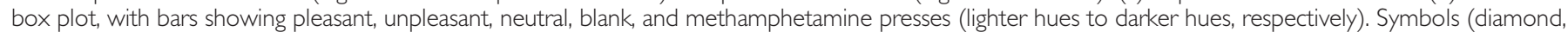

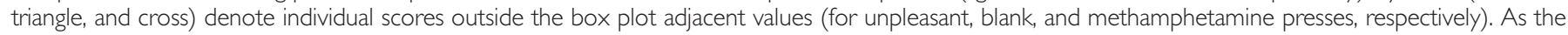

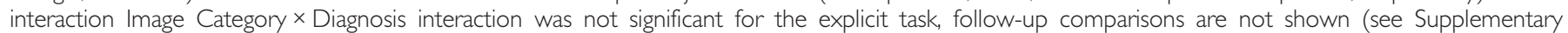

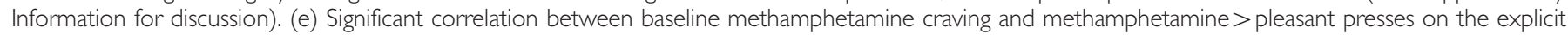
task in methamphetamine users. MA, methamphetamine users.

non-human content. Participants were instructed to choose their preferred images.

Probabilistic choice task. On each trial, participants used a button press to choose to view images on flipped-over cards, arranged in four decks. Immediately after selection from a particular deck, an image was revealed, and then covered the entire screen for passive viewing $(2000 \mathrm{msec})$ (Figure 1a). The images were arranged probabilistically: each deck contained 26 (of 30) images from a particular category (eg, pleasant), allowing images from other categories to be interspersed within each deck (two images from a secondary category, eg, methamphetamine; and one image from each of the two remaining categories). After selection from a particular deck eight times (corresponding to one task run), deck location of the four image categories shifted, and the participant's deck preference needed to be re-learned. We summed the number of cards selected per image category across four task runs.

Explicit choice task. Participants used continuous buttonpressing to choose between two side-by-side images from the same four categories (pleasant, unpleasant, neutral, methamphetamine) (Figure 1c). A fifth category, blank (black) screens, was also included. This blank-screen condition provided a comparison of the respective pictorial stimuli with nonstimuli (ie, choosing nothing), and has been part of the task as a second neutral condition since its development (Moeller et al, 2009). A choice enlarged the selected image to cover the screen, and participants could view that image for the $5000 \mathrm{msec}$ trial duration by continued button pressing; $500 \mathrm{msec}$ of non-response returned the side-by-side image display. After each trial, new images appeared. Each image category was represented an equal number of times throughout the task, and was displayed on the left or right side of the screen an equal number of times. The entire task comprised 70 trials (ie, seven repetitions in each of 10 image category pairs). Button pressing (working) for images was a design feature to simulate drug-seeking. We summed the total number of button presses executed for each picture category across the entire task while controlling for the number of presses overall (Moeller et al, 2009, 2010).

\section{PET Scanning}

Data were acquired using a Philips Gemini TF PET-CT (transverse and axial resolution $\mathrm{FWHM}=4.8 \mathrm{~mm}$ in the three-dimensional mode). Images were obtained with a $2-\mathrm{mm}$ voxel size (field of view $=128 \times 128 \times 90 \mathrm{~mm}^{3}$ ). A lowdose CT scan, performed before each PET scan, provided data for attenuation correction. Participants were placed on the scanning bed, in the supine position with the head positioned to avoid movement. For D1-type receptor scans, data were collected for $90 \mathrm{~min}$ after a bolus injection of $14.4 \pm 1.85 \mathrm{mCi}\left[{ }^{11} \mathrm{C}\right] \mathrm{NNC} 112$ (Abi-Dargham et al, 2000; Halldin et al, 1998) (specific activity: $4.9 \pm 5.61 \mathrm{Ci} / \mu \mathrm{mol})$; and 
D2-type receptor data were collected after a bolus injection of $5.0 \pm 0.37 \mathrm{mCi}\left[{ }^{18} \mathrm{~F}\right]$ fallypride (Mukherjee et al, 2002) (specific activity: $10.1 \pm 9.20 \mathrm{Ci} / \mu \mathrm{mol}$ ), in two 80 -min blocks with a 20-min intermission. Data were reconstructed with the row action maximum likelihood algorithm (RAMLA) implemented (Surti et al, 2007) for each 1-min frame. The time interval between scans with the two radiotracers in the methamphetamine users was $21.1 \pm 20.2$ days, although importantly this time interval did not correlate with choice behavior $(p>0.31)$ or any D1 $\mathrm{BP}_{\mathrm{ND}}$ measurements $(p>0.07)$ as defined below.

MRI scans were acquired to guide anatomical sampling (Siemens Sonata). T1-weighted, three-dimensional images, acquired with a magnetization-prepared rapid acquisition with gradient echo (MPRAGE) sequence (TR $=1900 \mathrm{~ms}$, $\mathrm{TE}=4.38 \mathrm{~ms}$, flip angle $=15^{\circ}$, field of view $=256 \times 256 \times 160$, 1 -mm voxels), were co-registered with PET images for definition of volumes-of-interest (VOIs) (details below).

Image processing. Reconstructed data from each $\left[{ }^{11} \mathrm{C}\right]$ NNC112 scan were averaged into 23 frames (four 1-min frames, three 2-min frames, 16 5-min frames). The reconstructed data from each $\left[{ }^{18} \mathrm{~F}\right]$ fallypride scan were combined into 16 images, each containing data averaged over $10 \mathrm{~min}$. After motion-correction using FSL MCFLIRT (Jenkinson et al, 2002), the PET images were co-registered to corresponding MRI scans using FSL FLIRT (Jenkinson and Smith, 2001). The primary VOI, the striatum, was divided into three functional bilateral subdivisions: limbic, associative, and sensorimotor (Mawlawi et al, 2001). More exploratory analyses were performed on other VOIs with appreciable $\mathrm{D} 2$-type $\mathrm{BP}_{\mathrm{ND}}$ for $\left[{ }^{18} \mathrm{~F}\right]$ fallypride (ie, amygdala, hippocampus, pallidum, thalamus, medial and lateral OFC,
ACC, and insula). VOIs, derived from the Harvard-Oxford atlases, were transformed into native space using FSL FNIRT, or were defined using FSL FIRST (Ardekani et al, 1995) (see Figure 2a).

Time-activity data within the VOIs were extracted from motion-corrected, co-registered PET images and imported into PMOD version 3.1 (PMOD Technologies Ltd., Zurich). The curves were fit using the simplified reference tissue model (SRTM) (Lammertsma and Hume, 1996) to estimate $k_{2}^{\prime}$, the rate parameter for transfer of the tracer from the reference region to plasma. The cerebellum was selected as a reference region because it has negligible concentrations of D1- and D2-type receptors and because the non-displaceable distribution volume of the radiotracer is similar to that of the tissue of interest: $K_{1} / k_{2}=K_{1}^{\prime} / k_{2}^{\prime}$, where the influx and efflux in the tissue of interest and the reference region are represented by $K_{1}$ and $k_{2}$, and $K_{1}^{\prime}$ and $k_{2}^{\prime}$, respectively. A volume-weighted average of $k_{2}^{\prime}$, estimated from high-activity regions (caudate and putamen), was computed. The time-activity curves were refit using SRTM2 (Wu and Carson, 2002) applying the computed $k_{2}^{\prime}$ values to all VOIs. $\mathrm{BP}_{\mathrm{ND}}$ was then calculated by subtracting 1.0 from the product of R1 (relative delivery of the tracer to the VOI compared with the reference region) and $k_{2}^{\prime} / k_{2 \mathrm{a}}$.

\section{Statistical Analyses}

Data from the probabilistic task were analyzed using a 4 (Image Category: pleasant, unpleasant, neutral, methamphetamine $\times 2$ (Diagnosis: methamphetamine user, control) mixed-model ANCOVA. Data from the explicit task were analyzed using a 5 (Image Category: pleasant, unpleasant, neutral, blank, methamphetamine) $\times 2$ (Diagnosis: a

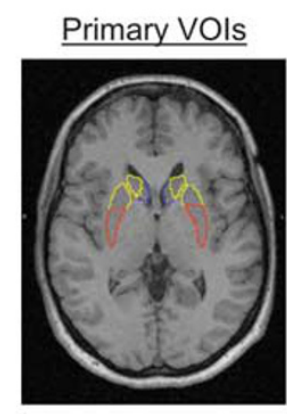

Exploratory VOIs

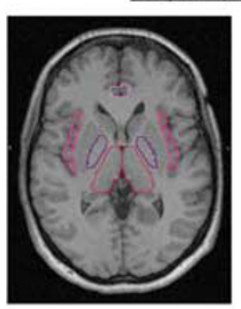

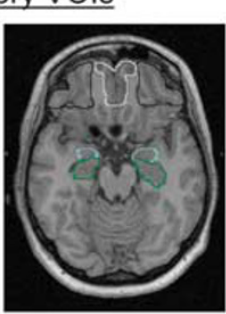

b

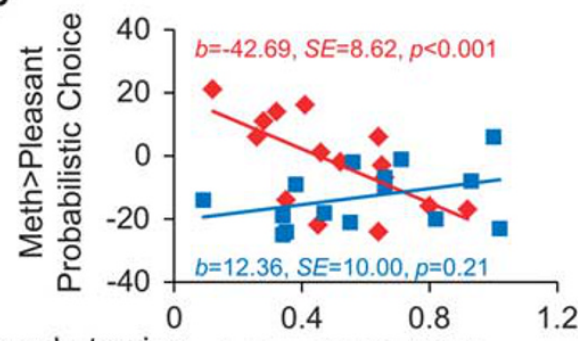

Methamphetamine Lateral OFC D2-Type

Control

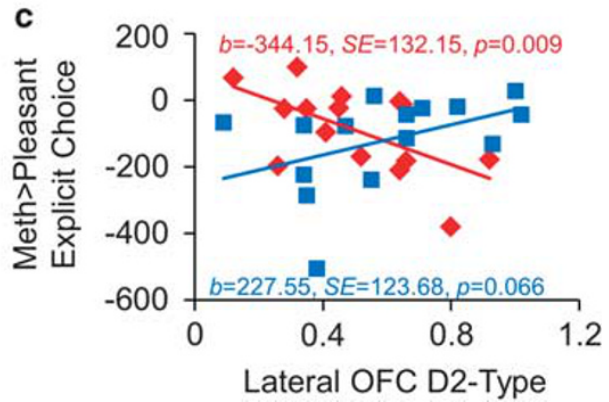

Figure 2 Associations between methamphetamine > pleasant choice and D2-type receptor availability. (a) Regions of interest. (b,c) Significant interactions between study group (Diagnosis) and D2-type receptor availability in the lateral orbitofrontal cortex (OFC) on methamphetamine $>$ pleasant choice as measured with the (b) probabilistic and (c) explicit tasks. For both tasks, negative relationships were observed in chronic methamphetamine users (the lower the receptor availability, the greater the methamphetamine-related choice), but not in healthy controls. 
methamphetamine user, control) mixed-model ANCOVA. Each ANCOVA covaried the total number of responses throughout the task (choices or presses, respectively). Follow-up comparisons were computed to localize the source of significant interactions. Age, sex, and smoking status were controlled in additional analyses to ensure that these variables did not drive the interactions. $P<0.05$ was considered significant.

We also tested correlations between drug-related choice (both tasks) and methamphetamine craving, administered before the choice tasks; and cumulative methamphetamine use, calculated as: (grams used) $\times$ (days/month used)/ $30 \times$ (months used), each considered significant at $p<0.013$ (four tests). For these analyses (and when testing associations with $\mathrm{BP}_{\mathrm{ND}}$, below), we focused a priori on the methamphetamine $>$ pleasant difference score, which is the most comparable to drug-choice administration studies. The direct drug $>$ pleasant contrast also has previously shown the greatest variance in choice behavior, which can then be tested for association with drug-relevant markers (eg, drug use outside the laboratory (Moeller et al, 2013a) or PET dopamine markers as tested here).

General linear models (GLMs) tested associations between methamphetamine $>$ pleasant image choice with D2- and D1-type receptor availability in the selected VOIs. For each model, we entered Diagnosis (categorical), $\mathrm{BP}_{\mathrm{ND}}$ in the respective VOI (continuous), and the interaction of the two to test association with methamphetamine $>$ pleasant choice, separately for each task. Because our goal was to examine for differential $\mathrm{BP}_{\mathrm{ND}}$ associations between methamphetamine users and controls, our primary interest was in testing the Diagnosis $\times \mathrm{BP}_{\mathrm{ND}}$ interaction (see the Supplementary Information for main effects of $\mathrm{BP}_{\mathrm{ND}}$ (ie, correlations across Diagnosis), and for exploratory analyses testing pleasant $>$ neutral choice, neither of which detract from our main findings). Analyses were conducted first without age, sex, and smoking status, and then controlling these variables. For all GLMs, a significance threshold of $p=0.004$ was established for the striatal VOIs $(3$ VOIs $\times 2$ PET measures $x$ 2 tasks $=12$ models), and a significance threshold of $p=0.002$ was established for all other VOIs (8 VOIs $\times 2$ PET measures $\times 2$ tasks $=32$ models). Owing to the presence of heteroscedasticity, particularly for the methamphetamine > pleasant contrast on the explicit task, the GLMs and correlations with drug use were conducted using WhiteHuber (robust) standard errors. D1-type $\mathrm{BP}_{\mathrm{ND}}$ data were unavailable for two methamphetamine users and five controls.

\section{RESULTS}

\section{Task Behavior}

Probabilistic task. Results of the mixed ANCOVA revealed a main effect of Image Category $(F(3,25)=16.43, p<0.001)$, such that all participants chose pleasant images most frequently, followed respectively by neutral, methamphetamine, and unpleasant images (for all pairwise comparisons, $p<0.05)$. Of greater interest, the Image Category $\times$ Diagnosis interaction reached significance $(F(3,25)=4.78$, $p=0.009$ ) (Figure 1b), which was robust to correction for age, gender, and smoking history $(p=0.025)$. Follow-up between-group analyses showed that, as expected, methamphetamine users selected more from methamphetamine decks than controls $(F(1,27)=12.75, p=0.001)$, whereas controls selected more from pleasant decks than methamphetamine users $(F(1,27)=10.68, p=0.003)$. These results are consistent with our hypotheses, and support our interest in the methamphetamine $>$ pleasant contrast. Follow-up within-group analyses showed that controls selected from methamphetamine decks less often than pleasant $(F(1,13)=$ 73.82, $p<0.001)$ and neutral $(F(1,13)=31.76, p<0.001)$ decks; methamphetamine users as a group, however, showed no differences in their selections for methamphetamine $v s$ either pleasant or neutral decks ( $p s>0.59$ ).

Explicit task. Results of the mixed Image Category $\times$ Diagnosis ANCOVA revealed a main effect of Image Category $(F(4,24)=46.58, p<0.001)$. All participants pressed the most for pleasant images, followed respectively by neutral images, blank and methamphetamine images (which did not differ), and finally unpleasant images (for all pairwise comparisons, except between blank and methamphetamine images, $p<0.05)$. The Image Category $\times$ Diagnosis ANCOVA was not significant $(F(4,24)=2.18, p=0.102)$ (Figure 1d), and so is not interpreted further here (for further discussion of this trend interaction, including a trend to press more for the methamphetamine images for the methamphetamine users than controls, see the Supplementary Information).

Task correlations with methamphetamine craving and use. The differential methamphetamine $>$ pleasant choice score on the explicit task was positively correlated with methamphetamine craving (the higher the craving, the higher the methamphetamine > pleasant button pressing: $b=-44.59, S E=13.87, z=-3.21, p=0.001$ ) (Figure 1e), and this correlation remained significant after correction for covariates $(p=0.003)$. Other correlations were not significant at the corrected threshold.

\section{Group Differences in $\mathbf{B P}_{\mathrm{ND}}$}

We have previously reported that, in a larger sample, methamphetamine users have lower $\mathrm{D} 2$-type $\mathrm{BP}_{\mathrm{ND}}$ than controls, and some of these same participants were included in this study. However, in this sample there were no Bonferroni-corrected differences for any VOI, for either D2type or D1-type receptor availability (all $t<2.09, p>0.051$ ) (Supplementary Table S1), although all data were in the expected direction (lower in methamphetamine users).

\section{PET-Choice Correlations}

Table 2 displays the zero-order correlations between $\mathrm{BP}_{\mathrm{ND}}$ and methamphetamine $>$ pleasant choice behavior, separately by Diagnosis. Table 3 displays the main GLM results. GLMs revealed Diagnosis $\times \mathrm{D} 2$-type $\mathrm{BP}_{\mathrm{ND}}$ interactions in the lateral OFC, for both the probabilistic $(b=-55.06$, $S E=12.97, z=-4.24, p=0.001)$, and explicit $(b=-571.70$, $S E=177.85, z=-3.21, p=0.001)$ tasks. That is, D2-type $\mathrm{BP}_{\mathrm{ND}}$ in the lateral OFC was negatively associated with methamphetamine $>$ pleasant choice in methamphetamine users (the lower the $\mathrm{BP}_{\mathrm{ND}}$, the higher the drug-related choice), but not in healthy controls (Figures $2 \mathrm{~b}$ and c). Both 
Table 2 Zero-Order Correlations Representing the Raw Associations between BP $\mathrm{ND}$ and Methamphetamine > Pleasant Choice Behavior Separately by Study Group (Diagnosis)

\begin{tabular}{|c|c|c|c|c|}
\hline & \multicolumn{2}{|c|}{$\begin{array}{l}\text { Probabilistic choice: } \\
\text { methamphetamine }>\text { pleasant }\end{array}$} & \multicolumn{2}{|c|}{$\begin{array}{c}\text { Explicit choice: } \\
\text { methamphetamine }>\text { pleasant }\end{array}$} \\
\hline & Controls $(r)$ & MA users (r) & Controls (r) & MA users $(r)$ \\
\hline \multicolumn{5}{|c|}{ D2-Type $B P_{N D}(15$ control, $15 \mathrm{MA})$} \\
\hline Limbic Striatum & -0.21 & -0.43 & 0.01 & -0.39 \\
\hline Associative Striatum & -0.35 & -0.37 & -0.16 & -0.45 \\
\hline Amygdala & -0.09 & -0.47 & 0.09 & -0.47 \\
\hline Hippocampus & 0.13 & $-0.54+$ & 0.09 & -0.43 \\
\hline Pallidum & -0.17 & -0.51 & 0.19 & -0.18 \\
\hline Thalamus & -0.06 & -0.49 & -0.20 & -0.47 \\
\hline Lateral OFC & 0.36 & $-0.65+$ & 0.44 & $-0.59+$ \\
\hline \multicolumn{5}{|c|}{ DI-Type $B P_{N D}(10$ control, $13 \mathrm{MA})$} \\
\hline Limbic Striatum & -0.30 & 0.36 & 0.17 & 0.14 \\
\hline Associative Striatum & -0.40 & 0.13 & 0.19 & 0.04 \\
\hline Sensorimotor Striatum & -0.31 & 0.11 & 0.10 & 0.03 \\
\hline Amygdala & -0.25 & 0.36 & 0.01 & 0.13 \\
\hline Hippocampus & 0.03 & 0.42 & -0.18 & 0.16 \\
\hline Pallidum & -0.28 & 0.29 & -0.19 & 0.28 \\
\hline Thalamus & 0.10 & 0.43 & -0.07 & 0.41 \\
\hline Lateral OFC & -0.58 & 0.34 & 0.26 & -0.08 \\
\hline
\end{tabular}

Note: ACC, anterior cingulate cortex; MA, methamphetamine, OFC, orbitofrontal cortex, for descriptive purposes, correlations at $p<0.05$ are marked by a cross.

interactions were robust to correction for covariates (Table 3). No additional Diagnosis $\times \mathrm{BP}_{\mathrm{ND}}$ interactions reached corrected significance, for either D2-type or D1 $\mathrm{BP}_{\mathrm{ND}}$, for any other VOI.

\section{DISCUSSION}

In this study, we examined behavior on two 'simulated' drugchoice tasks in individuals with methamphetamine use disorder and healthy controls, and tested association of such behavior with D2-type and D1-type receptor availability using PET. Behavioral analyses showed that both methamphetamine users and controls chose pleasant images most frequently on both tasks, with controls making more pleasant choices than methamphetamine users on the probabilistic task. The choice for methamphetamine images, however, depended on group membership. In methamphetamine users, the choice for methamphetamine images was comparable to neutral images; in controls, it was comparable to unpleasant images. Thus, methamphetamine users chose to view more drug-related images than controls on the probabilistic task (with a similar trend on the explicit task that requires verification in future studies; see the Supplementary Information). Although at first blush it may seem unexpected or problematic that the methamphetamine images appeared to be of modest value to methamphetamine users, our prior work in cocaine use disorder has similarly revealed high ambivalence about choosing drug-related images (Moeller et al, 2010). Importantly, the mean scores of methamphetamine choice might be less informative than the within-group variability of such choice, as the latter is crucial for testing association with relevant clinical markers as evidenced in our prior work (Moeller et al, 2013a) and in the rest of this study's findings.

The primary study finding was that higher methamphetamine $>$ pleasant choice on both tasks was associated with lower D2-type dopamine receptor availability in the lateral OFC, specifically in the methamphetamine users (indicated by significant Diagnosis $\times \mathrm{BP}_{\mathrm{ND}}$ interactions). This differential methamphetamine $>$ pleasant choice in methamphetamine users best approximates the drug-choice paradigms used in animal models and actively using humans (Moeller and Stoops, 2015), tapping into individual differences regarding the relative strength of choice for viewing 
Table 3 General Linear Model Results Testing for Diagnosis $\times \mathrm{BP}_{\mathrm{ND}}$ Interactions on Methamphetamine $>$ Pleasant Choice Behavior

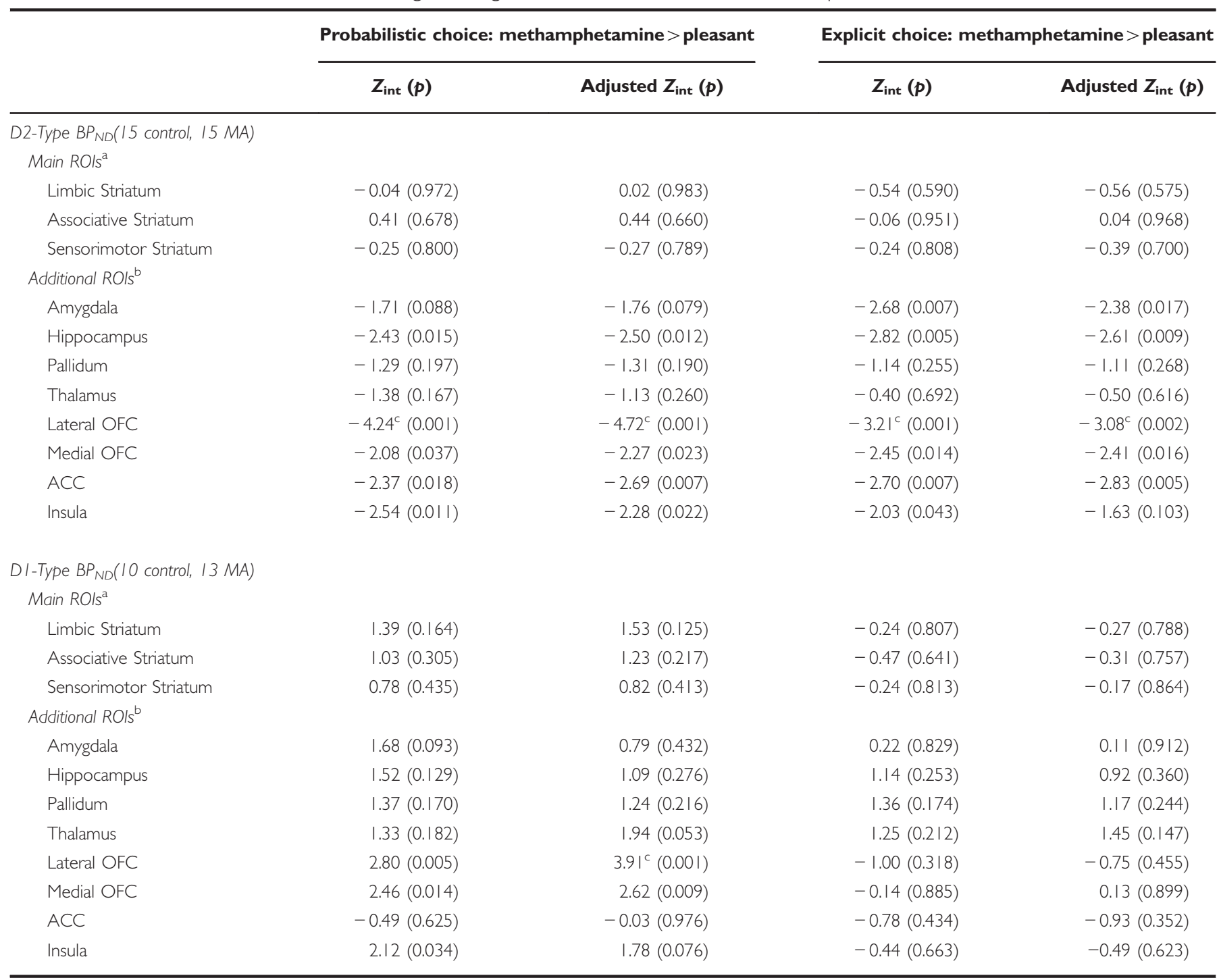

Abbreviations: ACC, anterior cingulate cortex, OFC, orbitofrontal cortex.

Note: predictors in the models include Diagnosis, $\mathrm{BP}_{\mathrm{ND}}$ and the Diagnosis $\times \mathrm{BP}_{\mathrm{ND}}$ interaction; $Z_{\text {int }}$ statistics correspond to the respective interaction effects (with corresponding $p$-values in parentheses); adjusted $Z_{\text {int }}$ statistics represent the same while controlling for age, gender, and smoking history (interactions with these covariates were not tested in the models).

aSignificance for main ROls was set at $p=0.004$.

bSignificance for additional ROls was set at $p=0.002$.

'Significant at these respective thresholds (using maximum likelihood estimation and robust standard errors).

methamphetamine images $v s$ affectively pleasant images. In this way, it becomes less likely that drugs and related cues are chosen for lack of other, more attractive options (Ahmed, 2010). In our study, higher methamphetamine $>$ pleasant choice on the explicit task in methamphetamine users was positively correlated with higher baseline craving (administered before the task). Of greater importance, methamphetamine users exhibited a consistent pattern of correlations between choice and $\mathrm{BP}_{\mathrm{ND}}$ on both tasks: the more that methamphetamine choice exceeded pleasant choice-or, alternatively, the closer methamphetamine choice came to exceeding pleasant choice-the lower was the D2-type $\mathrm{BP} \mathrm{P}_{\mathrm{ND}}$ in the lateral OFC.
A potential contribution of lateral OFC to drug-related choice behavior is not unexpected, given that this extended region contributes to multiple functions relevant to choice behavior and decision-making. For example, the OFC participates in salience/punishment (Berridge and Kringelbach, 2015), the updating of stimulus-reward associations (Noonan et al, 2011), and the evaluation of individual options independently of the others available (Rudebeck et al, 2013). Studies have also implicated the PFC including the OFC in modulating risky decision-making, and such modulation of PFC activation in turn has been correlated with D2-type receptor availability (in striatum) (Kohno et al, 2015); methamphetamine users, who had striatal D2-type receptor deficits, did not exhibit significant modulation of 
PFC activation by risk (Kohno et al, 2014). More recently, theory and evidence point to the OFC as constructing a latent or abstract representation about the task environment (Schuck et al, 2016), perhaps encoding the moment-tomoment value of task-relevant stimuli, choices, or actions based on current internal states (Rudebeck and Murray, 2014).

This study also had several unexpected or null findings. First, it was somewhat surprising that we did not find $\mathrm{BP}_{\mathrm{ND}}$ correlations in ventral or dorsal striatal regions, which a priori were main regions of interest. However, we note that the lack of a specific association in methamphetamine users between drug-related choice and striatal D2-type $\mathrm{BP}_{\mathrm{ND}}$ is consistent with an earlier study that examined choice for real cocaine administration over receiving an alternative reinforcer (merchandise voucher) (Martinez et al, 2004). Future studies could explore the possibility of differential connectivity between striatal and OFC regions. One hypothesis is that drug-biased choice could result from the interplay of drug-associated abnormalities in striatal reward circuitry and respective abnormalities in OFC functioning (London et al, 2000; Volkow and Fowler, 2000). Second, a previous study reported that striatal D1-type $\mathrm{BP}_{\mathrm{ND}}$ negatively correlated with the choice for real cocaine over merchandise vouchers (Martinez et al, 2009), whereas we did not find correlations between striatal D1-type $\mathrm{BP}_{\mathrm{ND}}$ and drug-related choice. Because we observed an interaction when examining an exploratory pleasant $>$ neutral contrast (Supplementary Information), the lack of effects for methamphetamine $>$ pleasant is not likely attributable to insufficient power. Rather, differences between the studies could involve the potential neurotoxicity of the two drugs (Schmitt and Reith, 2010), or recency of drug use, which may have affected choice in addition to the receptors assayed. In the prior cocaine studies, PET scans were performed following 14 days of abstinence, and the cocaine self-administration sessions were performed $2-3$ days after $\left[{ }^{11} \mathrm{C}\right]$ raclopride or $\left[{ }^{11} \mathrm{C}\right]$ NNC112 PET scans. In contrast, methamphetamine users studied here were more recently abstinent (4-7 days). Differences between hypothetical and real rewards could also be an influencing factor (Camerer and Mobbs, 2017).

For these reasons, and in conjunction with non-human animal studies showing that decision-making (eg, during risk) is modulated by both D1-type (Stopper et al, 2013) and D2-type receptors (Simon et al, 2011; Zalocusky et al, 2016), additional work is required to dissect the common and distinct roles of D1- vs D2-type receptors in drug-relevant decision-making in methamphetamine dependence. Nevertheless, our findings that correlations emerged between drug-choice and $\mathrm{BP}_{\mathrm{ND}}$ for $\mathrm{D} 2$-type receptors is largely consistent with available evidence specifying an important role of D2-type receptors in the pathophysiology of stimulant addiction (Ashok et al, 2017; London et al, 2015) -although it should be noted that prior studies have concentrated on D2 receptors in striatal regions. Furthermore, insofar as our drug-choice tasks are associated with OFC D2-type dopamine receptor availability and with methamphetamine craving, future studies can test the validity of these tasks in potentially interrogating OFC functioning in clinical contexts. Indeed, task associations with clinical outcomes previously have been observed in cocaine addiction (Moeller et al, 2013a), and deficient dopamine neurotransmission-albeit again in striatal regions-has predicted poorer treatment outcomes in cocaine and methamphetamine addiction (Martinez et al, 2011; Wang et al, 2012).

Limitations of this study include the following. First, the relatively small sample size limits statistical power, particularly in the D1-type BPND analyses. However, the pleasant $>$ neutral effect for D1-type $\mathrm{BP}_{\mathrm{ND}}$, reported in the Supplement, suggests that an effect similarly could have emerged for methamphetamine-related choice if it were there. Second, we acknowledge some inconsistency in behavior on the two choice tasks (ie, significant interaction for the probabilistic task as expected, but not for the explicit task). The lack of interaction for the explicit task may be partially attributable to the button pressing demands of the task. Although we controlled for the total number of presses, the variability in pressing was high. Third, there was a time gap between the choice tasks and D1-type (but not D2-type) assessments, although this choice-D1 time gap did not correlate with our main dependent variables and so is unlikely to have influenced these (null) results. Fourth, the ligands used in the present study have imperfect selectivity. Pharmacological blocking studies show that $\sim 25 \%$ of the $\left[{ }^{11} \mathrm{C}\right] \mathrm{NNC112}$ signal in the cortex represents binding to serotonin 5HT2A receptors (Ekelund et al, 2007; Slifstein et al, 2007), producing some contamination. Additionally, evidence that up to $20 \%$ of $\left[{ }^{18} \mathrm{~F}\right]$ fallypride may be bound to D3 dopamine receptors in vivo (Mukherjee et al, 2015) precludes making a definitive statement about either subtype.

In conclusion, D2-type, but not D1-type, receptor availability in the lateral OFC was correlated with, and possibly underlies, the choice to view drug-related images over affectively pleasant images in chronic methamphetamine users. By providing support for cortical involvement, these findings refine our understanding of a central research question in addiction concerning how dopamine deficits contribute to drug-biased decision-making. Results also support the important idea that a greater preference for drug rewards in the presence of similarly attractive non-drug rewards provides important markers of addiction severity and extent of underlying neurobiological abnormalities (Goldstein and Volkow, 2011).

\section{FUNDING AND DISCLOSURE}

This study was supported by grants from the National Institute on Drug Abuse (to SJM: K01DA037452; to ABK: F32DA039648; to RZG: R01DA041528, U01DA041174; supporting CLR: T32DA024635). The content is solely the responsibility of the authors and does not necessarily represent the official views of the National Institutes of Health. Additional support came from a Collaborative Research and Training Opportunities small grant award, sponsored by the Consortium in Brain Research (a collaboration between the Brain Research Institute of UCLA and the Friedman Brain Institute of the Icahn School of Medicine at Mount Sinai) (to SJM); and by and endowments from the Thomas P and Katherine K Pike Chair in Addiction Studies and the Marjorie M Greene Trust (to EDL). The authors declare no conflict of interest. 


\section{ACKNOWLEDGMENTS}

We thank Maritza Johnson for assistance with this study.

\section{REFERENCES}

Abi-Dargham A, Martinez D, Mawlawi O, Simpson N, Hwang DR, Slifstein $\mathrm{M}$ et al (2000). Measurement of striatal and extrastriatal dopamine D1 receptor binding potential with [11C]NNC 112 in humans: validation and reproducibility. J Cereb Blood Flow Metab 20: 225-243.

Ahmed SH (2010). Validation crisis in animal models of drug addiction: beyond non-disordered drug use toward drug addiction. Neurosci Biobehav Rev 35: 172-184.

Ardekani BA, Braun M, Hutton BF, Kanno I, Iida H (1995). A fully automatic multimodality image registration algorithm. J Comput Assist Tomogr 19: 615-623.

Ashok AH, Mizuno Y, Volkow ND, Howes OD (2017). Association of stimulant use with dopaminergic alterations in users of cocaine, amphetamine, or methamphetamine: a systematic review and meta-analysis. JAMA Psychiatry 74: 511-519.

Ballard ME, Mandelkern MA, Monterosso JR, Hsu E, Robertson CL, Ishibashi $\mathrm{K}$ et al (2015). Low dopamine D2/D3 receptor availability is associated with steep discounting of delayed rewards in methamphetamine dependence. Int J Neuropsychopharmacol 18: pyu119.

Bartra O, McGuire JT, Kable JW (2013). The valuation system: a coordinate-based meta-analysis of BOLD fMRI experiments examining neural correlates of subjective value. Neuroimage 76: 412-427.

Berridge KC, Kringelbach ML (2015). Pleasure systems in the brain. Neuron 86: 646-664.

Camerer C, Mobbs D (2017). Differences in behavior and brain activity during hypothetical and real choices. Trends Cogn Sci 21: 46-56.

Ekelund J, Slifstein M, Narendran R, Guillin O, Belani H, Guo NN et al (2007). In vivo DA D(1) receptor selectivity of NNC 112 and SCH 23390. Mol Imag Biol 9: 117-125.

First MB, Spitzer RL, Gibbon M, Williams J (1996). Williams J. Structured Clinical Interview for DSM-IV Axis I disorders - Patient Edition (SCID-I/P, Version 2.0). Biometrics Research Department, New York State Psychiatric Institute: New York, NY, USA.

Goldstein RZ, Volkow ND (2011). Dysfunction of the prefrontal cortex in addiction: neuroimaging findings and clinical implications. Nat Rev Neurosci 12: 652-669.

Greenwald MK, Ledgerwood DM, Lundahl LH, Steinmiller CL (2014). Effect of experimental analogs of contingency management treatment on cocaine seeking behavior. Drug Alcohol Depend 139: 164-168.

Halldin C, Foged C, Chou YH, Karlsson P, Swahn CG, Sandell J et al (1998). Carbon-11-NNC 112: a radioligand for PET examination of striatal and neocortical D1-dopamine receptors. J Nuclear Mede 39: 2061-2068.

Hogarth L, Chase HW (2011). Parallel goal-directed and habitual control of human drug-seeking: implications for dependence vulnerability. J Exp Psychol Animal Behav Processes 37: 261-276.

Jenkinson M, Bannister P, Brady M, Smith S (2002). Improved optimization for the robust and accurate linear registration and motion correction of brain images. Neuroimage 17: 825-841.

Jenkinson M, Smith S (2001). A global optimisation method for robust affine registration of brain images. Med Image Anal 5: 143-156.

Kohno M, Ghahremani DG, Morales AM, Robertson CL, Ishibashi K, Morgan AT et al (2015). Risk-taking behavior: dopamine D2/D3 receptors, feedback, and frontolimbic activity. Cereb Cortex 25: 236-245.
Kohno M, Morales AM, Ghahremani DG, Hellemann G, London ED (2014). Risky decision making, prefrontal cortex, and mesocorticolimbic functional connectivity in methamphetamine dependence. JAMA Psychiatry 71: 812-820.

Lammertsma AA, Hume SP (1996). Simplified reference tissue model for PET receptor studies. Neuroimage 4: 153-158.

Lang PJ, Bradley MM, Cuthbert BN (2008). International Affective Picture System (IAPS): Affective ratings of pictures and instruction manual. Technical Report A-8. University of Florida: Gainsville, FL, USA.

Lee B, London ED, Poldrack RA, Farahi J, Nacca A, Monterosso JR et al (2009). Striatal dopamine $\mathrm{d} 2 / \mathrm{d} 3$ receptor availability is reduced in methamphetamine dependence and is linked to impulsivity. J Neurosci 29: 14734-14740.

London ED, Ernst M, Grant S, Bonson K, Weinstein A (2000). Orbitofrontal cortex and human drug abuse: functional imaging. Cereb Cortex 10: 334-342.

London ED, Kohno M, Morales AM, Ballard ME (2015). Chronic methamphetamine abuse and corticostriatal deficits revealed by neuroimaging. Brain Res 1628: 174-185.

Martinez D, Broft A, Foltin RW, Slifstein M, Hwang DR, Huang Y et al (2004). Cocaine dependence and $\mathrm{d} 2$ receptor availability in the functional subdivisions of the striatum: relationship with cocaine-seeking behavior. Neuropsychopharmacology 29: 1190-1202.

Martinez D, Carpenter KM, Liu F, Slifstein M, Broft A, Friedman $\mathrm{AC}$ et al (2011). Imaging dopamine transmission in cocaine dependence: link between neurochemistry and response to treatment. Am J Psychiatry 168: 634-641.

Martinez D, Narendran R, Foltin RW, Slifstein M, Hwang DR, Broft A et al (2007). Amphetamine-induced dopamine release: markedly blunted in cocaine dependence and predictive of the choice to self-administer cocaine. Am J Psychiatry 164: 622-629.

Martinez D, Slifstein M, Narendran R, Foltin RW, Broft A, Hwang $\mathrm{DR}$ et al (2009). Dopamine D1 receptors in cocaine dependence measured with PET and the choice to self-administer cocaine. Neuropsychopharmacology 34: 1774-1782.

Mawlawi O, Martinez D, Slifstein M, Broft A, Chatterjee R, Hwang DR et al (2001). Imaging human mesolimbic dopamine transmission with positron emission tomography: I. Accuracy and precision of $\mathrm{D}(2)$ receptor parameter measurements in ventral striatum. J Cereb Blood Flow Metab 21: 1034-1057.

Moeller SJ, Beebe-Wang N, Woicik PA, Konova AB, Maloney T, Goldstein RZ (2013a). Choice to view cocaine images predicts concurrent and prospective drug use in cocaine addiction. Drug Alcohol Depend 130: 178-185.

Moeller SJ, Maloney T, Parvaz MA, Alia-Klein N, Woicik PA, Telang $\mathrm{F}$ et al (2010). Impaired insight in cocaine addiction: laboratory evidence and effects on cocaine-seeking behaviour. Brain 133: 1484-1493.

Moeller SJ, Maloney T, Parvaz MA, Dunning JP, Alia-Klein N, Woicik PA et al (2009). Enhanced choice for viewing cocaine pictures in cocaine addiction. Biol Psychiatry 66: 169-176.

Moeller SJ, Parvaz MA, Shumay E, Beebe-Wang N, Konova AB, Alia-Klein $\mathrm{N}$ et al (2013b). Gene $\mathrm{x}$ abstinence effects on drug cue reactivity in addiction: multimodal evidence. J Neurosci 33: 10027-10036.

Moeller SJ, Stoops WW (2015). Cocaine choice procedures in animals, humans, and treatment-seekers: can we bridge the divide? Pharmacol Biochem Behav 138: 133-141.

Mukherjee J, Christian BT, Dunigan KA, Shi B, Narayanan TK, Satter M et al (2002). Brain imaging of 18 F-fallypride in normal volunteers: blood analysis, distribution, test-retest studies, and preliminary assessment of sensitivity to aging effects on dopamine D-2/D-3 receptors. Synapse 46: 170-188.

Mukherjee J, Constantinescu CC, Hoang AT, Jerjian T, Majji D, Pan ML (2015). Dopamine D3 receptor binding of (18)F- 
fallypride: Evaluation using in vitro and in vivo PET imaging studies. Synapse 69: 577-591.

Noonan MP, Mars RB, Rushworth MF (2011). Distinct roles of three frontal cortical areas in reward-guided behavior. J Neurosci 31: 14399-14412.

Padoa-Schioppa C (2011). Neurobiology of economic choice: a good-based model. Annu Rev Neurosci 34: 333-359.

Rudebeck PH, Mitz AR, Chacko RV, Murray EA (2013). Effects of amygdala lesions on reward-value coding in orbital and medial prefrontal cortex. Neuron 80: 1519-1531.

Rudebeck PH, Murray EA (2014). The orbitofrontal oracle: cortical mechanisms for the prediction and evaluation of specific behavioral outcomes. Neuron 84: 1143-1156.

Schmitt KC, Reith ME (2010). Regulation of the dopamine transporter: aspects relevant to psychostimulant drugs of abuse. Ann N Y Acad Sci 1187: 316-340.

Schuck NW, Cai MB, Wilson RC, Niv Y (2016). Human orbitofrontal cortex represents a cognitive map of state space. Neuron 91: 1402-1412.

Simon NW, Montgomery KS, Beas BS, Mitchell MR, LaSarge CL, Mendez IA et al (2011). Dopaminergic modulation of risky decision-making. J Neurosci 31: 17460-17470.
Slifstein M, Kegeles LS, Gonzales R, Frankle WG, Xu X, Laruelle M et al (2007). [11C]NNC 112 selectivity for dopamine D1 and serotonin 5-HT(2 A) receptors: a PET study in healthy human subjects. J Cereb Blood Flow Metab 27: 1733-1741.

Stopper CM, Khayambashi S, Floresco SB (2013). Receptor-specific modulation of risk-based decision making by nucleus accumbens dopamine. Neuropsychopharmacology 38: 715-728.

Surti S, Kuhn A, Werner ME, Perkins AE, Kolthammer J, Karp JS (2007). Performance of Philips Gemini TF PET/CT scanner with special consideration for its time-of-flight imaging capabilities. J Nuclear Med 48: 471-480.

Volkow ND, Fowler JS (2000). Addiction, a disease of compulsion and drive: involvement of the orbitofrontal cortex. Cereb Cortex 10: $318-325$.

Wang GJ, Smith L, Volkow ND, Telang F, Logan J, Tomasi D et al (2012). Decreased dopamine activity predicts relapse in methamphetamine abusers. Mol Psychiatry 17: 918-925.

$\mathrm{Wu}$ Y, Carson RE (2002). Noise reduction in the simplified reference tissue model for neuroreceptor functional imaging. J Cereb Blood Flow Metab 22: 1440-1452.

Zalocusky KA, Ramakrishnan C, Lerner TN, Davidson TJ, Knutson B, Deisseroth K (2016). Nucleus accumbens D2R cells signal prior outcomes and control risky decision-making. Nature 531: 642-646.

Supplementary Information accompanies the paper on the Neuropsychopharmacology website (http://www.nature.com/npp) 